\title{
Series Solution of the Multispecies Lotka-Volterra Equations by Means of the Homotopy Analysis Method
}

\author{
A. Sami Bataineh, M. S. M. Noorani, and I. Hashim \\ School of Mathematical Sciences, Faculty of Science and Tecnology, Universiti Kebangsaan Malaysia, \\ 43600 Bangi, Selangor, Malaysia
}

Correspondence should be addressed to I. Hashim, ishak_h@ukm.my

Received 9 May 2008; Accepted 2 July 2008

Recommended by Yong Zhou

The time evolution of the multispecies Lotka-Volterra system is investigated by the homotopy analysis method (HAM). The continuous solution for the nonlinear system is given, which provides a convenient and straightforward approach to calculate the dynamics of the system. The HAM continuous solution generated by polynomial base functions is of comparable accuracy to the purely numerical fourth-order Runge-Kutta method. The convergence theorem for the three-dimensional case is also given.

Copyright (C) 2008 A. Sami Bataineh et al. This is an open access article distributed under the Creative Commons Attribution License, which permits unrestricted use, distribution, and reproduction in any medium, provided the original work is properly cited.

\section{Introduction}

The simplest model of predator-prey interactions was developed independently by Lotka [1] and Volterra [2]. The classical two-dimensional Lotka-Volterra equation is given by

$$
\begin{aligned}
& \frac{\mathrm{d} y_{1}}{\mathrm{~d} t}=a y_{1}-b y_{1} y_{2}, \\
& \frac{\mathrm{d} y_{2}}{\mathrm{~d} t}=-c y_{2}+b y_{1} y_{2} .
\end{aligned}
$$

System (1.1) has been one of the most studied models for a two-dimensional dynamical system. The generalized $n$-dimensional Lotka-Volterra equations are given by (cf. $[3,4])$

$$
\frac{\mathrm{d} y_{i}(t)}{\mathrm{d} t}=y_{i}(t)\left(b_{i}+\sum_{j=1}^{n} a_{i j} y_{j}(t)\right), \quad i=1,2, \ldots, n,
$$


subject to the initial conditions

$$
y_{i}(0)=c_{i}, \quad i=1,2, \ldots, n,
$$

where the $a^{\prime}$ s, $b^{\prime}$ s, and $c^{\prime}$ s are constants. System (1.2)-(1.3) has a wide applicability to a variety of different physical [5], chemical [6], and biological problems [7].

In the study of nonlinear systems of differential equations such as the Lotka-Volterra equation, analytical solutions are usually unknown. In this case, in order to analyze the behavior of the system, one usually resorts to numerical integration techniques, such as the Runge-Kutta method [8], or perturbation techniques [9]. The problem with purely numerical technique like the Runge-Kutta method is that it does not give a functional form of the solution to the problem at hand, which is often useful if we need to scrutinize the solution in detail. Perturbation techniques depend on the existence of small or large parameters in the nonlinear problems.

The homotopy analysis method (HAM), initially proposed by Liao in his Ph.D. thesis [10], is a powerful analytic method for nonlinear problems. A systematic and clear exposition on HAM is given in [11]. In recent years, this method has been successfully employed to solve many types of nonlinear problems in science and engineering [12-29]. More recently, Bataineh et al. [30-33] employed the standard HAM to solve some problems in engineering sciences. HAM yields rapidly convergent series solutions in most cases, usually only a few iterations leading to very accurate solutions. Very recently, Bataineh et al. [34, 35] presented two modifications of HAM (MHAM) to solve systems of second-order BVPs and homogeneous or nonhomogeneous differential equations with constant or variable coefficients. HAM and its modifications contain a certain auxiliary parameter $\hbar$, which provides us with a simple way to adjust and control the convergence region and rate of convergence of the series solution. Moreover, by means of the so-called $\hbar$-curve, it is easy to find the valid regions of $\hbar$ to gain a convergent series solution. Thus, through HAM, explicit analytic solutions of nonlinear problems are possible. Apart from providing us with a functional form of the solution to the nonlinear problem, another advantage of HAM is that the method is valid for equations without small or large parameters like the Lotka-Volterra equation.

In this paper, we will employ HAM to obtain series solutions to the multispecies LotkaVolterra competition models which are governed by a system of nonlinear ordinary differential equations. The HAM gives continuous solution which is of comparable accuracy to purely numerical method like the classical fourth-order Runge-Kutta method (RK4). The convergence theorem for the three-dimensional case is also given.

\section{HAM for system of ODEs}

We consider the following system of differential equations:

$$
N_{i}\left[y_{i}(t)\right]=g_{i}(t), \quad i=1,2, \ldots, n,
$$

where $N_{i}$ are nonlinear operators, $t$ denotes the independent variable, $y_{i}(t)$ are unknown functions, and $g_{i}(t)$ are known analytic functions representing the nonhomogeneous terms. If $g_{i}(t)=0,(2.1)$ reduces to the homogeneous equation. By means of generalizing the traditional homotopy method [11], we construct the so-called zeroth-order deformation equation:

$$
(1-q) L\left[\phi_{i}(t ; q)-y_{i, 0}(t)\right]=q \hbar\left\{N_{i}\left[\phi_{i}(t ; q)\right]-g_{i}(t)\right\},
$$


where $q \in[0,1]$ is an embedding parameter, $\hbar$ is a nonzero auxiliary function, $L$ is an auxiliary linear operator, $y_{i, 0}(t)$ are the initial guesses of $y_{i}(t)$, and $\phi_{i}(t ; q)$ are unknown functions. It is important to note that one has great freedom to choose the auxiliary objects such as $\hbar$ and $L$ in HAM. Obviously, when $q=0$ and $q=1$, both

$$
\phi_{i}(t ; 0)=y_{i, 0}(t), \quad \phi_{i}(t ; 1)=y_{i}(t)
$$

hold. Thus, as $q$ increases from 0 to 1 , the solutions $\phi_{i}(t ; q)$ vary from the initial guesses $y_{i, 0}(t)$ to the solutions $y_{i}(t)$. Expanding $\phi_{i}(t ; q)$ in Taylor series with respect to $q$, one has

$$
\phi_{i}(t ; q)=y_{i, 0}(t)+\sum_{m=1}^{+\infty} y_{i, m}(t) q^{m}
$$

where

$$
y_{i, m}=\left.\frac{1}{m !} \frac{\partial^{m} \phi_{i}(t ; q)}{\partial q^{m}}\right|_{q=0} .
$$

If the auxiliary linear operator, the initial guesses, the auxiliary parameters $\hbar$, and the auxiliary functions are so properly chosen, then the series (2.4) converges at $q=1$ and

$$
\phi_{i}(t ; 1)=y_{i}(t)=y_{i, 0}(t)+\sum_{m=1}^{+\infty} y_{i, m}(t)
$$

which must be one of the solutions of the original nonlinear equation, as proved by [11]. As $\hbar=-1,(2.2)$ becomes

$$
(1-q) L\left[\phi_{i}(t ; q)-y_{i, 0}(t)\right]+q\left\{N_{i}\left[\phi_{i}(t ; q)\right]-g_{i}(t)\right\}=0,
$$

which is used mostly in the HPM [36].

According to (2.5), the governing equations can be deduced from the zeroth-order deformation equations (2.2). Define the vectors

$$
\vec{y}_{i, n}=\left\{y_{i, 0}(t), y_{i, 1}(t), \ldots, y_{i, n}(t)\right\} .
$$

Differentiating (2.2) $m$ times with respect to the embedding parameter $q$, then setting $q=0$, and finally dividing them by $m$ !, we have the so-called $m$ th-order deformation equation

$$
L\left[y_{i, m}(t)-x_{m} y_{i, m-1}(t)\right]=\hbar R_{i, m}\left(\vec{y}_{i, m-1}\right),
$$

where

$$
\begin{gathered}
R_{i, m}\left(\vec{y}_{i, m-1}\right)=\left.\frac{1}{(m-1) !} \frac{\partial^{m-1}\left\{N_{i}\left[\phi_{i}(t ; q)\right]-g_{i}(t)\right\}}{\partial q^{m-1}}\right|_{q=0}, \\
x_{m}= \begin{cases}0, & m \leq 1, \\
1, & m>1 .\end{cases}
\end{gathered}
$$

It should be emphasized that $y_{i, m}(t)(m \geq 1)$ is governed by the linear equation (2.9) with the linear initial/boundary conditions that come from the original problem, which can be easily solved by symbolic computation softwares such as Maple and Mathematica. 


\section{Applications of HAM}

We will next obtain series solutions to the one-, two-, and three-dimensional cases of (1.2)-(1.3) by HAM separately. We assume, in this paper, that the solution to (1.2)-(1.3) can be expressed by the set of polynomial base functions:

$$
\left\{t^{m} \mid m=1,2,3, \ldots\right\}
$$

from which we have

$$
y(t)=\sum_{m=0}^{\infty} A_{m} t^{m}
$$

where $A_{m}$ are coefficients to be determined. This provides us with the so-called rule of solution expression; that is, the solution of (1.2) must be expressed in the same form as (3.2).

\subsection{One-dimensional case}

Consider the 1D case of (1.2), known as the Verhulst equation,

$$
\frac{\mathrm{d} y}{\mathrm{~d} t}=b y-a y^{2}
$$

where $a$ and $b$ are positive constants. The exact solution of (3.3) can be found by direct integration and is given by

$$
y(t)=\frac{b \mathrm{e}^{b t}}{(b+a y(t)) / y(0)-a \mathrm{e}^{b t}} \quad \text { for } b \neq 0 .
$$

For definiteness, we will assume the following initial condition:

$$
y(0)=0.1
$$

To solve (3.3) by HAM with the initial condition (3.5) and with $b=1$ and $a=3$, we first choose the initial approximation

$$
y_{0}(t)=0.1
$$

and the linear operator

$$
L[\phi(t ; q)]=\frac{\partial \phi(t ; q)}{\partial t}
$$

with the property

$$
L[c]=0,
$$


where $c$ is an integral constant. Furthermore, (3.3) suggests that we define the nonlinear operator as

$$
N[\phi(t ; q)]=\frac{\partial \phi(t ; q)}{\partial t}-b \phi(t ; q)+a \phi^{2}(t ; q)
$$

Using the above definition, we construct the zeroth-order deformation equation as in (2.2), and the $m$ th-order deformation equation for $m \geq 1$ is as in (2.9), subject to the initial condition

$$
y_{m}(0)=0,
$$

where

$$
R_{m}\left[\vec{y}_{m-1}\right]=y_{m-1}^{\prime}(t)-b y_{m-1}(t)+a \sum_{i=0}^{m-1} y_{i}(t) y_{m-1-i}(t) .
$$

Now, the solution of the $m$ th-order deformation equation (2.9) becomes

$$
y_{m}(t)=x_{m} y_{m-1}(t)+\hbar \int_{0}^{t} R_{m}\left(\vec{y}_{m-1}\right) d \tau+c,
$$

where the integration of constant $c$ is determined by the initial condition (3.10). We now successively obtain

$$
\begin{aligned}
y_{1}(t) & =-\frac{7}{100} \hbar t \\
y_{2}(t) & =-\frac{7}{100} \hbar t-\frac{7}{100} \hbar^{2} t+\frac{14}{1000} \hbar^{2} t^{2} \\
& \vdots
\end{aligned}
$$

In general, the analytic solution of (1.2) via the polynomial base functions is given by

$$
y(t)=\sum_{m=1}^{+\infty} d_{m}(\hbar) t^{m}
$$

\subsection{Two-dimensional case}

Now we apply HAM to solve the 2D version of (1.2):

$$
\begin{aligned}
& \frac{\mathrm{d} y_{1}(t)}{\mathrm{d} t}=y_{1}(t)\left[b_{1}+a_{11} y_{1}(t)+a_{12} y_{2}(t)\right], \\
& \frac{\mathrm{d} y_{2}(t)}{\mathrm{d} t}=y_{2}(t)\left[b_{2}+a_{21} y_{1}(t)+a_{22} y_{2}(t)\right],
\end{aligned}
$$

where $a^{\prime}$ s and $b^{\prime}$ s are constants and subject to the initial conditions

$$
y_{1}(0)=4, \quad y_{2}(0)=10
$$


According to HAM, the initial approximations of system (3.15)-(3.16) are

$$
y_{1,0}(t)=4, \quad y_{2,0}(0)=10,
$$

and the auxiliary linear operators for $i=1,2$ are

$$
L\left[\phi_{i}(t ; q)\right]=\frac{\partial \phi_{i}(t ; q)}{\partial t}, \quad i=1,2,
$$

with the property

$$
L\left[c_{i}\right]=0
$$

and the nonlinear operators

$$
\begin{aligned}
& N_{1}\left[\phi_{i}(t ; q)\right]=\frac{\partial \phi_{1}(t ; q)}{\partial t}-\phi_{1}(t ; q)\left(b_{1}+a_{11} \phi_{1}(t ; q)+a_{12} \phi_{2}(t ; q)\right), \\
& N_{2}\left[\phi_{i}(t ; q)\right]=\frac{\partial \phi_{2}(t ; q)}{\partial t}-\phi_{2}(t, q)\left(b_{2}+a_{21} \phi_{1}(t ; q)+a_{22} \phi_{2}(t ; q)\right) .
\end{aligned}
$$

Again, using the above definition, we construct the zeroth-order deformation equation as in (2.2), and the $m$ th-order deformation equation for $m \geq 1$ is as in (2.9), subject to the initial condition

$$
y_{i, m}(0)=0,
$$

where

$$
\begin{aligned}
& R_{1, m}\left[\vec{y}_{i, m-1}(t)\right]=y_{1, m-1}^{\prime}(t)-b_{1} y_{1, m-1}(t)-a_{11} \sum_{i=0}^{m-1} y_{1, i}(t) y_{1, m-1-i}(t)-a_{12} \sum_{i=0}^{m-1} y_{1, i}(t) y_{2, m-1-i}(t), \\
& R_{2, m}\left[\vec{y}_{i, m-1}(t)\right]=y_{2, m-1}^{\prime}(t)-b_{2} y_{2, m-1}(t)-a_{21} \sum_{i=0}^{m-1} y_{2, i}(t) y_{1, m-1-i}(t)-a_{22} \sum_{i=0}^{m-1} y_{2, i}(t) y_{2, m-1-i}(t),
\end{aligned}
$$

where the prime denotes differentiation with respect to the similarity variable $t$. Now, the solution of the $m$ th-order deformation equation (2.9) for $m \geq 1$ and $i=1,2$ is given by

$$
y_{i, m}(t)=x_{m} y_{i, m-1}(t)+\hbar \int_{0}^{t} R_{1, m}\left(\vec{y}_{i, m-1}\right) d \tau+c_{i}
$$

where the integration constants $c_{i}(i=1,2)$ are determined by the initial condition (3.21). Thereafter, we successively obtain

$$
\begin{array}{cl}
y_{1,1}(t)=-0.3296 \hbar t, & y_{1,2}(t)=-0.3296 \hbar t-0.3296 \hbar^{2} t+0.011063 \hbar^{2} t^{2}, \\
y_{2,1}(t)=-0.664 \hbar t, & y_{2,2}(t)=-0.664 \hbar t-0.664 \hbar^{2} t+0.0172416 \hbar^{2} t^{2}
\end{array}
$$

and so forth. Thus, the analytic solution of (3.15)-(3.16) has the general form

$$
y_{i}(t)=\sum_{m=1}^{+\infty} a_{i, m}(\hbar) t^{m}
$$




\subsection{Three-dimensional case}

Finally, we apply HAM to solve the 3D version of (1.2):

$$
\begin{aligned}
& \frac{\mathrm{d} y_{1}(t)}{\mathrm{d} t}=y_{1}(t)\left(b_{1}+a_{11} y_{1}(t)+a_{12} y_{2}(t)+a_{13} y_{3}(t)\right), \\
& \frac{\mathrm{d} y_{2}(t)}{\mathrm{d} t}=y_{2}(t)\left(b_{2}+a_{21} y_{1}(t)+a_{22} y_{2}(t)+a_{23} y_{3}(t)\right), \\
& \frac{\mathrm{d} y_{3}(t)}{\mathrm{d} t}=y_{3}(t)\left(b_{3}+a_{31} y_{1}(t)+a_{32} y_{2}(t)+a_{33} y_{3}(t)\right),
\end{aligned}
$$

with the initial conditions

$$
y_{1,0}(0)=0.2, \quad y_{2,0}(0)=0.3, \quad y_{3,0}(0)=0.5,
$$

where the $a^{\prime}$ s and $b^{\prime}$ s are constants.

According to HAM, the initial approximations of (3.26) are

$$
y_{1,0}(t)=0.2, \quad y_{2,0}(t)=0.3, \quad y_{3,0}(t)=0.5,
$$

and the auxiliary linear operators are as in (3.18) with the property (3.19), where $c_{i}(i=1,2,3)$ are constants of integrations. In a similar way as in the previous systems, we obtain the $m$ thorder deformation equation (2.9), where

$$
\begin{aligned}
R_{1, m}\left[\vec{y}_{i, m-1}(t)\right]= & y_{1, m-1}^{\prime}(t)-b_{1} y_{1, m-1}(t)-a_{11} \sum_{i=0}^{m-1} y_{1, i}(t) y_{1, m-1-i}(t) \\
& -a_{12} \sum_{i=0}^{m-1} y_{1, i}(t) y_{2, m-1-i}(t)-a_{13} \sum_{i=0}^{m-1} y_{1, i}(t) y_{3, m-1-i}(t), \\
R_{2, m}\left[\vec{y}_{i, m-1}(t)\right]= & y_{2, m-1}^{\prime}(t)-b_{2} y_{2, m-1}(t)-a_{21} \sum_{i=0}^{m-1} y_{2, i}(t) y_{1, m-1-i}(t) \\
& -a_{22} \sum_{i=0}^{m-1} y_{2, i}(t) y_{2, m-1-i}(t)-a_{23} \sum_{i=0}^{m-1} y_{2, i}(t) y_{3, m-1-i}(t), \\
R_{3, m}\left[\vec{y}_{i, m-1}(t)\right]= & y_{3, m-1}^{\prime}(t)-b_{3} y_{3, m-1}(t)-a_{31} \sum_{i=0}^{m-1} y_{3, i}(t) y_{1, m-1-i}(t) \\
& -a_{32} \sum_{i=0}^{m-1} y_{3, i}(t) y_{2, m-1-i}(t)-a_{33} \sum_{i=0}^{m-1} y_{3, i}(t) y_{3, m-1-i}(t),
\end{aligned}
$$

subject to the initial condition

$$
y_{i, m}(0)=0 \text {. }
$$


Following similar procedure as in the previous section, we find the first two terms of the series solution:

$$
\begin{array}{ll}
y_{1,1}(t)=-0.144 \hbar t, & y_{1,2}(t)=-0.144 \hbar t-0.144 \hbar^{2} t+0.0333 \hbar^{2} t^{2}, \\
y_{2,1}(t)=-0.189 \hbar t, & y_{2,2}(t)=-0.189 \hbar t-0.189 \hbar^{2} t+0.02565 \hbar^{2} t^{2}, \\
y_{3,1}(t)=-0.225 \hbar t, & y_{3,2}(t)=-0.225 \hbar t-0.225 \hbar^{2} t-0.01395 \hbar^{2} t^{2} .
\end{array}
$$

Then, the solution expression via the polynomial base functions can be written as in (3.25).

We will next give a convergence theorem for the HAM series solution of the 3D version of (1.2).

\subsubsection{Convergence theorem}

As long as the series $y_{i}(t)=y_{i, 0}(t)+\sum_{m=1}^{+\infty} y_{i, m}(t)$ converges, where $y_{i, m}(t)$ is governed by (2.9) under the definitions (3.29), (3.30), and (2.11), it must be the solution of (3.26).

Proof. If the series is convergent, we can write for $i=1,2,3$ that

$$
S_{i}=\sum_{m=0}^{+\infty} y_{i, m}(t)
$$

and there hold

$$
\lim _{n \rightarrow+\infty} y_{1, n}(t)=0, \quad \lim _{n \rightarrow+\infty} y_{2, n}=0, \quad \lim _{n \rightarrow+\infty} y_{3, n}=0
$$

From (2.9) and by using the definitions (2.11) and (3.18), we then have

$$
\begin{aligned}
\hbar \sum_{m=1}^{+\infty} R_{i, m}\left(\vec{y}_{i, m-1}\right) & =\sum_{m=1}^{+\infty} L\left[y_{i, m}(t)-x_{m} y_{i, m-1}(t)\right] \\
& =\lim _{n \rightarrow+\infty} \sum_{m=1}^{n} L\left[y_{i, m}(t)-x_{m} y_{i, m-1}(t)\right] \\
& =L\left[\lim _{n \rightarrow+\infty} \sum_{m=1}^{n} y_{i, m}(t)-x_{m} y_{i, m-1}(t)\right] \\
& =L\left[\lim _{n \rightarrow+\infty} y_{i, n}(t)\right] \\
& =0,
\end{aligned}
$$

which gives, since $\hbar \neq 0$,

$$
\sum_{n=1}^{+\infty} R_{i, n}\left(\vec{y}_{i, n-1}\right)=0
$$


On the other hand, substituting (3.29), respectively, into the above expressions and simplifying them, we obtain

$$
\begin{aligned}
& \sum_{m=1}^{+\infty} R_{1, m}\left(\vec{y}_{i, m-1}\right)=\sum_{m=1}^{+\infty}\left[y_{1, m-1}^{\prime}(t)-b_{1} y_{1, m-1}(t)-a_{11} \sum_{i=0}^{m-1} y_{1, i}(t) y_{2, m-1-i}(t)\right. \\
& \left.-a_{12} \sum_{i=0}^{m-1} y_{1, i}(t) y_{2, m-1-i}(t)-a_{13} \sum_{i=0}^{m-1} y_{1, i}(t) y_{3, m-1-i}(t)\right] \\
& =\sum_{m=1}^{+\infty} y_{1, m-1}^{\prime}(t)-b_{1} y_{1, m-1}(t)-a_{11} \sum_{m=1}^{+\infty} \sum_{i=0}^{m-1} y_{1, i}(t) y_{1, m-1-i}(t) \\
& -a_{12} \sum_{m=1}^{+\infty} \sum_{i=0}^{m-1} y_{1, i}(t) y_{2, m-1-i}(t)-a_{13} \sum_{m=1}^{+\infty} \sum_{i=0}^{m-1} y_{1, i}(t) y_{3, m-1-i}(t) \\
& =\sum_{m=1}^{+\infty} y_{1, m-1}^{\prime}(t)-b_{1} y_{1, m-1}(t)-a_{11} \sum_{i=0}^{+\infty} \sum_{m=i+1}^{+\infty} y_{1, i}(t) y_{1, m-1-i}(t) \\
& -a_{12} \sum_{i=0}^{+\infty} \sum_{m=i+1}^{+\infty} y_{1, i}(t) y_{2, m-1-i}(t)-a_{13} \sum_{i=0}^{+\infty} \sum_{m=i+1}^{+\infty} y_{1, i}(t) y_{3, m-1-i}(t) \\
& =\sum_{m=0}^{+\infty} y_{1, m}^{\prime}(t)-b_{1} y_{1, m}(t)-a_{11} \sum_{i=0}^{+\infty} y_{1, i}(t) \sum_{j=0}^{+\infty} y_{1, j}(t) \\
& -a_{12} \sum_{i=0}^{+\infty} y_{1, i}(t) \sum_{j=0}^{+\infty} y_{2, j}(t)-a_{13} \sum_{i=0}^{+\infty} y_{1, i}(t) \sum_{j=0}^{+\infty} y_{3, j}(t) \\
& =S_{1}^{\prime}(t)-S_{1}(t)\left[b_{1}+a_{11} S_{1}(t)+a_{12} S_{2}(t)+a_{13} S_{3}(t)\right]=0, \\
& \sum_{m=1}^{+\infty} R_{2, m}\left(\vec{y}_{i, m-1}\right)=\sum_{m=1}^{+\infty}\left[y_{2, m-1}^{\prime}(t)-b_{2} y_{2, m-1}(t)-a_{21} \sum_{i=0}^{m-1} y_{2, i}(t) y_{1, m-1-i}(t)\right. \\
& \left.-a_{22} \sum_{i=0}^{m-1} y_{2, i}(t) y_{2, m-1-i}(t)-a_{23} \sum_{i=0}^{m-1} y_{2, i}(t) y_{3, m-1-i}(t)\right] \\
& =\sum_{m=1}^{+\infty} y_{2, m-1}^{\prime}(t)-b_{2} y_{2, m-1}(t)-a_{21} \sum_{m=1}^{+\infty} \sum_{i=0}^{m-1} y_{2, i}(t) y_{1, m-1-i}(t) \\
& -a_{22} \sum_{m=1}^{+\infty} \sum_{i=0}^{m-1} y_{2, i}(t) y_{2, m-1-i}(t)-a_{23} \sum_{m=1}^{+\infty} \sum_{i=0}^{m-1} y_{2, i}(t) y_{3, m-1-i}(t) \\
& =\sum_{m=1}^{+\infty} y_{2, m-1}^{\prime}(t)-b_{2} y_{2, m-1}(t)-a_{21} \sum_{i=0}^{+\infty} \sum_{m=i+1}^{+\infty} y_{2, i}(t) y_{1, m-1-i}(t) \\
& -a_{22} \sum_{i=0}^{+\infty} \sum_{m=i+1}^{+\infty} y_{2, i}(t) y_{2, m-1-i}(t)-a_{23} \sum_{i=0}^{+\infty} \sum_{m=i+1}^{+\infty} y_{2, i}(t) y_{3, m-1-i}(t)
\end{aligned}
$$




$$
\begin{aligned}
= & \sum_{m=0}^{+\infty} y_{2, m}^{\prime}(t)-b_{2} y_{2, m}(t)-a_{21} \sum_{i=0}^{+\infty} y_{2, i}(t) \sum_{j=0}^{+\infty} y_{1, j}(t) \\
& -a_{22} \sum_{i=0}^{+\infty} y_{2, i}(t) \sum_{j=0}^{+\infty} y_{2, j}(t)-a_{23} \sum_{i=0}^{+\infty} y_{2, i}(t) \sum_{j=0}^{+\infty} y_{3, j}(t) \\
= & S_{2}^{\prime}(t)-S_{2}(t)\left[b_{2}+a_{21} S_{1}(t)+a_{12} S_{2}(t)+a_{13} S_{3}(t)\right]=0 \\
\sum_{m=1}^{+\infty} R_{3, m}\left(\vec{y}_{i, m-1}\right)= & \sum_{m=1}^{+\infty}\left[y_{3, m-1}^{\prime}(t)-b_{3} y_{3, m-1}(t)-a_{31} \sum_{i=0}^{m-1} y_{3, i}(t) y_{1, m-1-i}(t)\right. \\
= & \sum_{m=1}^{+\infty} y_{3, m-1}^{\prime}(t)-b_{3} y_{3, m-1}(t)-a_{31} \sum_{m=1}^{+\infty} \sum_{i=0}^{m-1} y_{3, i}(t) y_{1, m-1-i}(t) \\
& -a_{32} \sum_{m=1}^{+\infty} \sum_{i=0}^{m-1} y_{3, i}(t) y_{2, m-1-i}(t)-a_{33} \sum_{m=1}^{+\infty} \sum_{i=0}^{m-1} y_{3, i}(t) y_{3, m-1-i}(t) \\
= & \sum_{m=1}^{+\infty} y_{3, m-1}^{\prime}(t)-b_{3} y_{3, m-1}(t)-a_{31} \sum_{i=0}^{+\infty} \sum_{m=i+1}^{+\infty} y_{3, i}(t) y_{1, m-1-i}(t) \\
& -a_{32} \sum_{i=0}^{+\infty} \sum_{m=i+1}^{+\infty} y_{3, i}(t) y_{2, m-1}(t) y_{3, m-1-i}(t)-a_{33} \sum_{i=0}^{+\infty} \sum_{m=i+1}^{+\infty} y_{3, i}(t) y_{3, m-1-i}(t) \\
= & \sum_{m=0}^{+\infty} y_{3, m}^{\prime}(t)-b_{3} y_{3, m}(t)-a_{31} \sum_{i=0}^{+\infty} y_{3, i}(t) \sum_{j=0}^{+\infty} y_{1, j}(t) \\
& -a_{32} \sum_{i=0}^{+\infty} y_{3, i}(t) \sum_{j=0}^{+\infty} y_{2, j}(t)-a_{33} \sum_{i=0}^{+\infty} y_{3, i}(t)\left[b_{3}+a_{31} \sum_{j=0}^{+\infty} y_{1, j}(t)+a_{32} S_{2}(t)+a_{33} S_{3}(t)\right]=0 \\
& \\
& \\
&
\end{aligned}
$$

From the initial conditions (3.28) and (3.30), there hold

$$
\begin{aligned}
& S_{1}(0)=y_{1,0}(0)+\sum_{m=1}^{+\infty} y_{1, m}(0)=0.2, \\
& S_{2}(0)=y_{2,0}(0)+\sum_{m=1}^{+\infty} y_{2, m}(0)=0.3, \\
& S_{3}(0)=y_{3,0}(0)+\sum_{m=1}^{+\infty} y_{3, m}(0)=0.5 .
\end{aligned}
$$

So, $S_{i}(t)$ satisfy (3.26), and are therefore solutions of the 3D version of (1.2) with the initial condition (3.30). This ends the proof. 


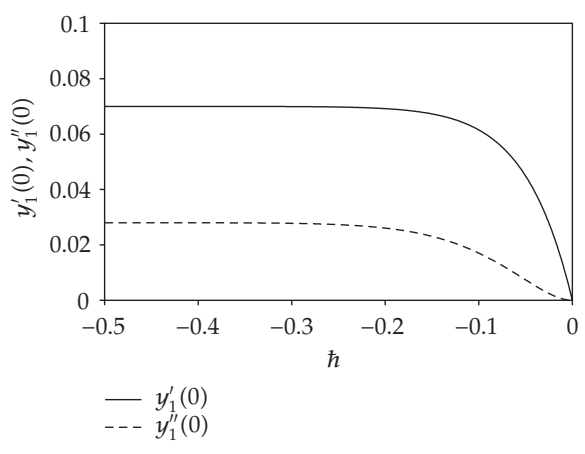

(a)

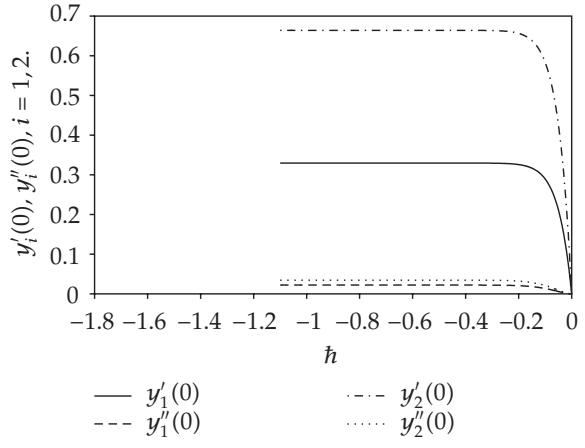

(b)

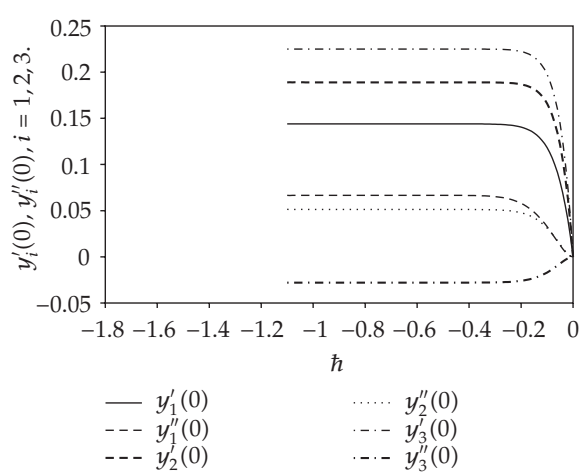

(c)

Figure 1: The $\hbar$-curves obtained from the 20th-order HAM approximation solutions of (a) (3.3); (b) (3.15); (c) (3.26).

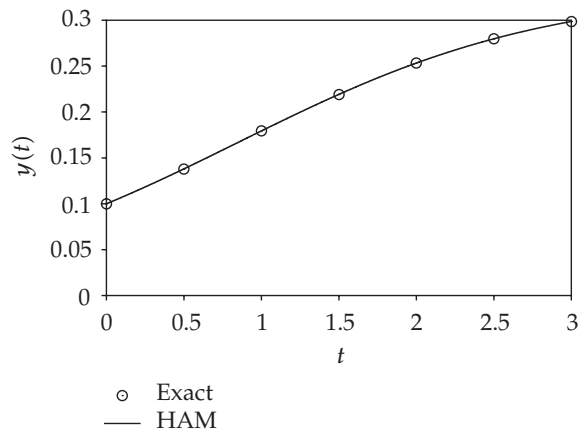

Figure 2: The 61th-order HAM solution of (3.3) with $\hbar=-0.25$ versus RK4 ( $\Delta t=0.001)$ solution for the 1D case.

\section{Results and discussions}

The series solutions of (1.2) given by HAM contain the auxiliary parameter $\hbar$. The validity of the method is based on such an assumption that the series (2.4) converges at $q=1$. It is the auxiliary parameter $\hbar$ which ensures that this assumption can be satisfied. In general, by means 


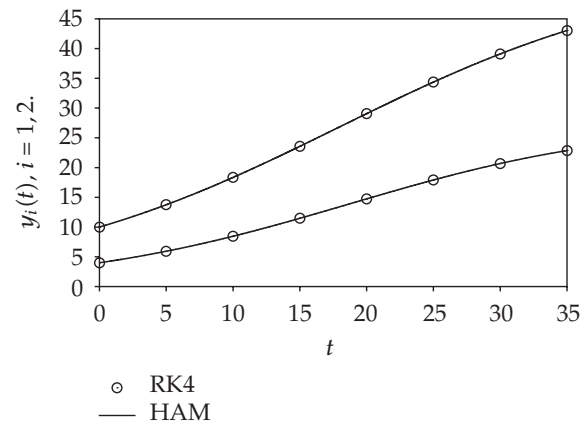

Figure 3: The 31th-order HAM solution of (3.15) with $\hbar=-0.8$ versus RK4 $(\Delta t=0.001)$ solution for the 2D case.

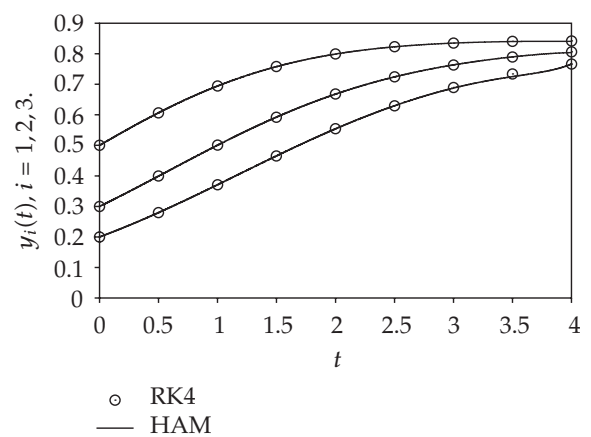

Figure 4: The 41th-order HAM solution of (3.26) with $\hbar=-0.6$ versus RK4 ( $\Delta t=0.001)$ solution for the 3D case.

of the so-called $\hbar$-curve, it is straightforward to choose a proper value of $\hbar$ which ensures that the solution series is convergent. Figure 1 shows the $\hbar$-curves of $1 \mathrm{D}, 2 \mathrm{D}$, and $3 \mathrm{D}$ obtained from the 20th-order HAM approximation solutions of (1.2). From these figures, the valid regions of $\hbar$ correspond to the line segments nearly parallel to the horizontal axis. In Figures 2, 3, and 4, it is demonstrated that the HAM solutions, taking $\hbar=-0.25, \hbar=-0.8$, and $\hbar=-0.6$, agree very well with the solutions obtained by the classical fourth-order Runge-Kutta method at the step size $\Delta t=0.001$.

\section{Conclusions}

In this paper, the homotopy analysis method (HAM) was applied to solve the Lotka-Volterra equations. Polynomial base functions were found to give very good accuracy of HAM solutions for the Lotka-Volterra equations. The HAM gives continuous solution which is of comparable accuracy to purely numerical method like the classical fourth-order Runge-Kutta method (RK4). This is convenient for practical applications with minimum requirements on calculation and computation. The convergence theorem for the three-dimensional case is also given. We remark that the validity of the HAM series solutions can be enhanced by finding more terms and/or using the Padé technique. The functional form of the solution would be useful in the study of the stability of the system. 


\section{Acknowledgments}

The financial support received from MOSTI Sciencefund, Grant no. 04-01-02-SF0177, and from the Academy of Sciences Malaysia under SAGA Grant no. STGL-011-2006 (P24c) is gratefully acknowledged. The referee with the constructive comments is also acknowledged.

\section{References}

[1] A. J. Lotka, Elements of Physical Biology, Williams \& Wilkins, Baltimore, Md, USA, 1925.

[2] V. Volterra, "Variazioni e fluttuazioni del numero d'individui in specie animali conviventi," Memorie della Reale Accademia dei Lincei, vol. 2, pp. 31-113, 1926.

[3] R. M. May and W. J. Leonard, "Nonlinear aspects of competition between three species," SIAM Journal on Applied Mathematics, vol. 29, no. 2, pp. 243-253, 1975.

[4] E. C. Pielou, An Introduction to Mathematical Ecology, Wiley-Interscience, New York, NY, USA, 1969.

[5] V. W. Noonburg, "A neural network modeled by an adaptive Lotka-Volterra system," SIAM Journal on Applied Mathematics, vol. 49, no. 6, pp. 1779-1792, 1989.

[6] Z. Noszticzius, E. Noszticzius, and Z. A. Schelly, "Use of ion-selective electrodes for monitoring oscillating reactions. 2. Potential response of bromide- iodide-selective electrodes in slow corrosive processes. Disproportionation of bromous and iodous acids. A Lotka-Volterra model for the halate driven oscillators," Journal of Physical Chemistry, vol. 87, no. 3, pp. 510-524, 1983.

[7] K.-I. Tainaka, "Stationary pattern of vortices or strings in biological systems: lattice version of the Lotka-Volterra model," Physical Review Letters, vol. 63, no. 24, pp. 2688-2691, 1989.

[8] A. M. Stuart and A. R. Humphries, Dynamical Systems and Numerical Analysis, vol. 2 of Cambridge Monographs on Applied and Computational Mathematics, Cambridge University Press, Cambridge, UK, 1996.

[9] A. H. Nayfeh, Perturbation Methods, Wiley Classics Library, John Wiley \& Sons, New York, NY, USA, 2000.

[10] S. J. Liao, The proposed homotopy analysis techniques for the solution of nonlinear problems, Ph.D. dissertation, Shanghai Jiao Tong University, Shanghai, China, 1992.

[11] S. J. Liao, Beyond Perturbation: Introduction to the Homotopy Analysis Method, vol. 2 of CRC Series: Modern Mechanics and Mathematics, Chapman \& Hall/CRC, Boca Raton, Fla, USA, 2004.

[12] S. J. Liao, "An approximate solution technique not depending on small parameters: a special example," International Journal of Non-Linear Mechanics, vol. 30, no. 3, pp. 371-380, 1995.

[13] S. J. Liao, "A kind of approximate solution technique which does not depend upon small parametersII: an application in fluid mechanics," International Journal of Non-Linear Mechanics, vol. 32, no. 5, pp. 815-822, 1997.

[14] S. J. Liao, "An explicit, totally analytic approximate solution for Blasius' viscous flow problems," International Journal of Non-Linear Mechanics, vol. 34, no. 4, pp. 759-778, 1999.

[15] S. J. Liao, "On the homotopy analysis method for nonlinear problems," Applied Mathematics and Computation, vol. 147, no. 2, pp. 499-513, 2004.

[16] S. J. Liao and I. Pop, "Explicit analytic solution for similarity boundary layer equations," International Journal of Heat and Mass Transfer, vol. 47, no. 1, pp. 75-85, 2004.

[17] S. J. Liao, "Comparison between the homotopy analysis method and homotopy perturbation method," Applied Mathematics and Computation, vol. 169, no. 2, pp. 1186-1194, 2005.

[18] S. J. Liao, "A new branch of solutions of boundary-layer flows over an impermeable stretched plate," International Journal of Heat and Mass Transfer, vol. 48, no. 12, pp. 2529-2539, 2005.

[19] S. J. Liao and Y. Tan, "A general approach to obtain series solutions of nonlinear differential equations," Studies in Applied Mathematics, vol. 119, no. 4, pp. 297-354, 2007.

[20] S. J. Liao, "Notes on the homotopy analysis method: some definitions and theorems," Communications in Nonlinear Science and Numerical Simulation. In press.

[21] M. Ayub, A. Rasheed, and T. Hayat, "Exact flow of a third grade fluid past a porous plate using homotopy analysis method," International Journal of Engineering Science, vol. 41, no. 18, pp. 2091-2103, 2003.

[22] T. Hayat, M. Khan, and S. Asghar, "Homotopy analysis of MHD flows of an Oldroyd 8-constant fluid," Acta Mechanica, vol. 168, no. 3-4, pp. 213-232, 2004. 
[23] T. Hayat and M. Khan, "Homotopy solutions for a generalized second-grade fluid past a porous plate," Nonlinear Dynamics, vol. 42, no. 4, pp. 395-405, 2005.

[24] M. Sajid and T. Hayat, "Comparison of HAM and HPM methods in nonlinear heat conduction and convection equations," Nonlinear Analysis: Real World Applications. In press.

[25] I. Hashim, O. Abdulaziz, and S. Momani, "Homotopy analysis method for fractional IVPs," Communications in Nonlinear Science and Numerical Simulation. In press.

[26] M. S. H. Chowdhury, I. Hashim, and O. Abdulaziz, "Comparison of homotopy analysis method and homotopy-perturbation method for purely nonlinear fin-type problems," Communications in Nonlinear Science and Numerical Simulation. In press.

[27] Y. Tan and S. Abbasbandy, "Homotopy analysis method for quadratic Riccati differential equation," Communications in Nonlinear Science and Numerical Simulation, vol. 13, no. 3, pp. 539-546, 2008.

[28] S. Abbasbandy, "The application of homotopy analysis method to nonlinear equations arising in heat transfer," Physics Letters A, vol. 360, no. 1, pp. 109-113, 2006.

[29] S. Abbasbandy, "The application of homotopy analysis method to solve a generalized HirotaSatsuma coupled KdV equation," Physics Letters A, vol. 361, no. 6, pp. 478-483, 2007.

[30] A. S. Bataineh, M. S. M. Noorani, and I. Hashim, "Solving systems of ODEs by homotopy analysis method," Communications in Nonlinear Science and Numerical Simulation, vol. 13, no. 10, pp. 2060-2070, 2008.

[31] A. S. Bataineh, M. S. M. Noorani, and I. Hashim, "Solutions of time-dependent Emden-fowler type equations by homotopy analysis method," Physics Letters A, vol. 371, no. 1-2, pp. 72-82, 2007.

[32] A. S. Bataineh, M. S. M. Noorani, and I. Hashim, "Approximate solutions of singular two-point BVPs by modified homotopy analysis method," Physics Letters A, vol. 372, no. 22, pp. 4062-4066, 2008.

[33] A. S. Bataineh, M. S. M. Noorani, and I. Hashim, "Approximate analytical solutions of systems of PDEs by homotopy analysis method," Computers $\mathcal{E}$ Mathematics with Applications, vol. 55, no. 12, pp. 2913-2923, 2008.

[34] A. S. Bataineh, M. S. M. Noorani, and I. Hashim, "Modified homotopy analysis method for solving systems of second-order BVPs," Communications in Nonlinear Science and Numerical Simulation. In press.

[35] A.S. Bataineh, M. S. M. Noorani, and I. Hashim, "On a new reliable modification of homotopy analysis method," Communications in Nonlinear Science and Numerical Simulation. In press.

[36] J.-H. He, "Homotopy perturbation method: a new nonlinear analytical technique," Applied Mathematics and Computation, vol. 135, no. 1, pp. 73-79, 2003. 


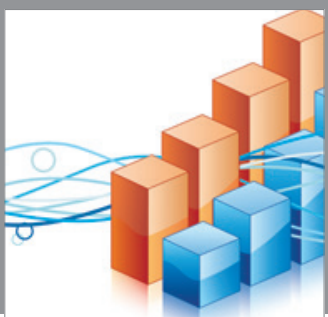

Advances in

Operations Research

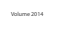

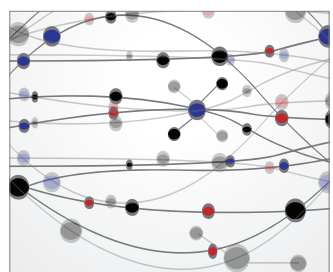

\section{The Scientific} World Journal
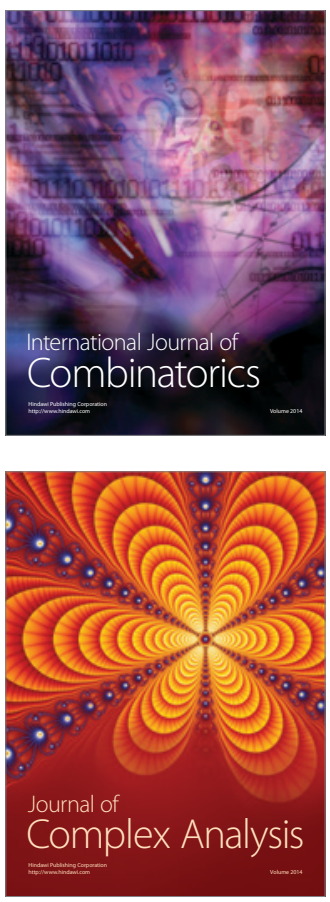

International Journal of

Mathematics and

Mathematical

Sciences
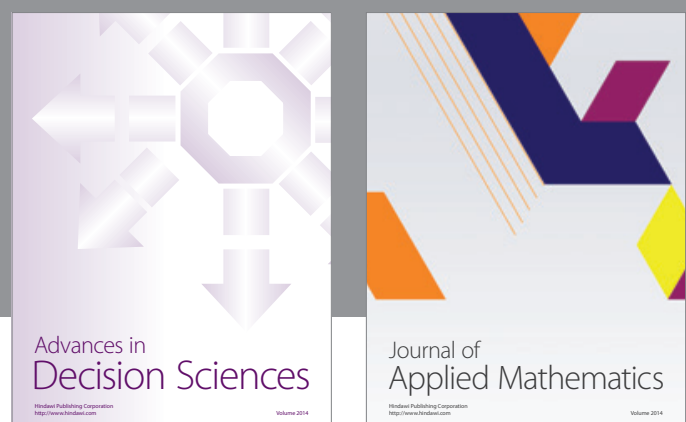

Journal of

Applied Mathematics
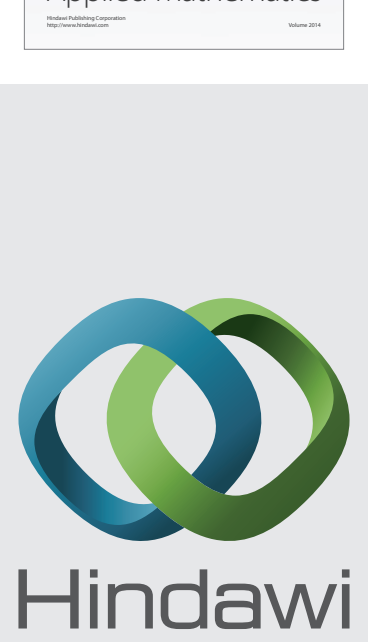

Submit your manuscripts at http://www.hindawi.com
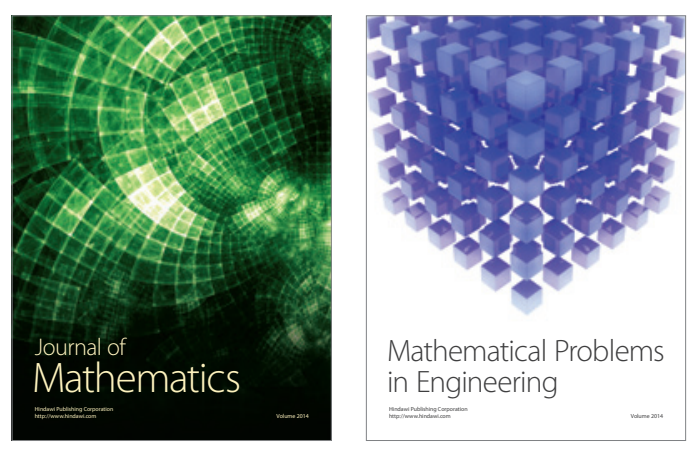

Mathematical Problems in Engineering
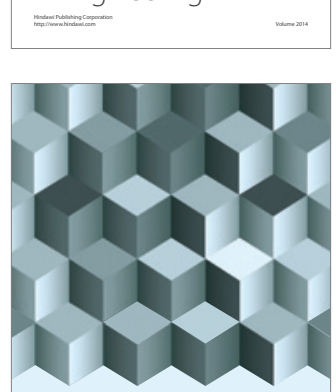

Journal of

Function Spaces
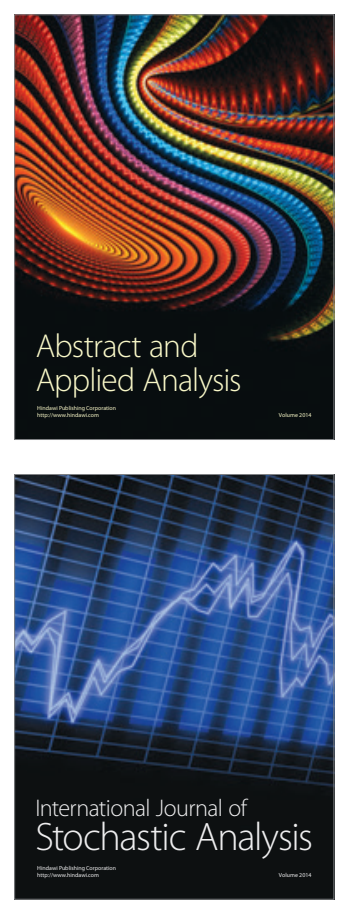

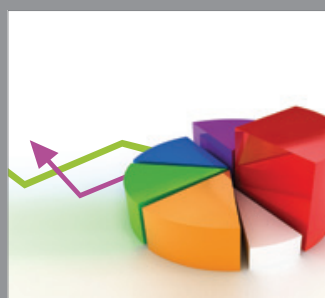

ournal of

Probability and Statistics

Promensencen
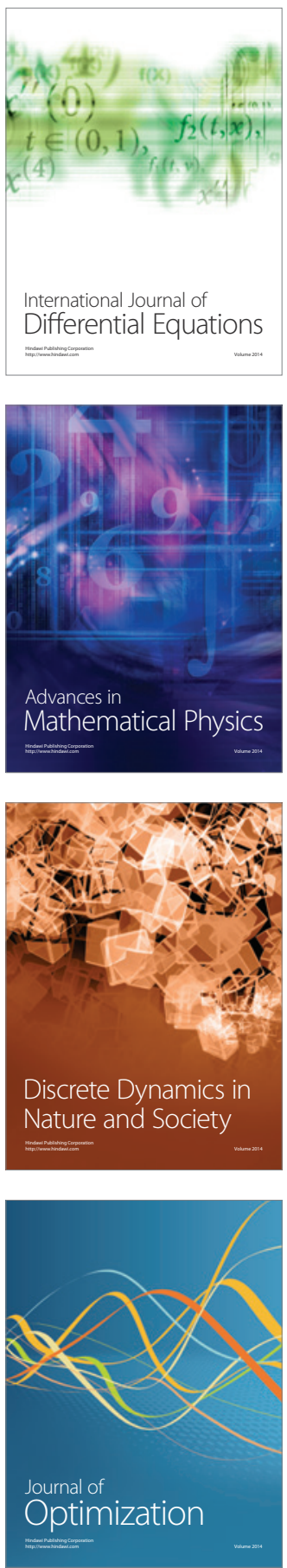